\title{
Desenvolvimento Preliminar de um Algoritmo de Reconhecimento Facial em Tempo Real através de Redes Neurais Convolucionais
}

\author{
Diana Rocha, João Pereira, Maria Melo, Bruno C. Bispo e Pedro M. Rodrigues
}

\begin{abstract}
Resumo-Este trabalho apresenta os resultados preliminares do desenvolvimento de um algoritmo de reconhecimento facial em tempo real através de redes neurais convolucionais (CNNs). Elementos faciais, nomeadamente, rosto, boca, nariz e olhos, são detectados pelo algoritmo de Viola-Jones. Cada elemento facial é utilizado para treinar uma CNN. Os resultados de treinamento mostram uma acurácia de identificação de $100 \%$. Testes em tempo real demonstram necessidade de aprimoramento. A base de imagens será futuramente ampliada para realização de um rigoroso procedimento de treinamento e teste do algoritmo.

Palavras-Chave - Reconhecimento facial, tempo real, rede neural convolucional, algoritmo de Viola-Jones.

Abstract-This work presents the preliminary results of the development of a real-time facial recognition algorithm through convolutional neural networks (CNNs). Facial elements, namely, face, mouth, nose and eyes, are detected by the Viola-Jones algorithm. Each facial element is used to train a CNN. The training results show $100 \%$ identification accuracy. Real-time tests demonstrate a need for improvement. The image base will be expanded in the future to carry out a rigorous procedure for training and testing the algorithm.
\end{abstract}

Keywords-Face recognition, real time, convolutional neural network, Viola-Jones algorithm.

\section{Introdução}

A face é o principal elemento para a identificação de uma pessoa. O reconhecimento facial é crucial em muitas aplicações como, por exemplo, ciber segurança e monitorização de pessoas em tempo real [1]. Este trabalho apresenta os resultados preliminares do desenvolvimento de um algoritmo para identificar, em tempo real, indivíduos através de uma rede neural convolucional (CNN) e elementos faciais, nomeadamente, rosto, boca, nariz e olhos.

\section{Metodologia}

O método aplicado pode ser dividido em 3 etapas: aquisição digital de imagem e pré-processamento; segmentação e extração de elementos faciais; reconhecimento em tempo real. A Figura 1 resume a metodologia utilizada.

\section{A. Aquisição e pré-processamento de imagem}

A primeira etapa constituiu na aquisição de 300 imagens do rosto de cada um dos 3 indivíduos, que participaram deste estudo inicial, através de uma webcam. As imagens na escala RGB foram convertidas para a escala de cinza. Imagens do nariz, boca e olhos foram extraídas de cada imagem do rosto.

Diana Rocha, João Pereira, Maria Melo, e Pedro M. Rodrigues, Universidade Católica Portuguesa, CBQF - Centro de Biotecnologia e Química Fina Laboratório Associado, Escola Superior de Biotecnologia, Porto, Portugal, email: prodrigues@ porto.ucp.pt; Bruno C. Bispo, Departamento de Engenharia Elétrica e Eletrônica, Universidade Federal de Santa Catarina, FlorianópolisSC, Brasil, e-mail: bruno.bispo@ufsc.br.

\section{B. Segmentação e extração de elementos faciais}

Para detectar os elementos faciais em tempo real, nomeadamente, rosto, boca, nariz e olhos, utilizou-se o algoritmo ViolaJones. Este algoritmo tem a capacidade de processar imagens a uma alta velocidade e de apresentar altas taxas de detecção [2], [3]. Apesar de amplamente utilizado para detectar rostos, ele pode ser utilizado para detectar qualquer tipo de objeto. O algoritmo é composto por 4 etapas [2], [3]: características Haar; imagens integrais; treinamento adaboost; e classificador em cascata. Estas etapas são brevemente descritas abaixo. Maiores detalhes podem ser encontrados em [2], [3].

1) Caraterísticas Haar: Características, ou kernels, constituídas de regiões retangulares integralmente formadas por pixels brancos ou pretos, onde as regiões possuem o mesmo tamanho e são adjacentes horizontal ou verticalmente. Possuem esse nome por lembrarem as funções base da Haar. Características com até 4 regiões são usadas. Aplicadas a uma sub-janela da imagem, o valor resultante é a diferença entre a soma dos pixels dentro das regiões claras e a soma dos pixels dentro das regiões escuras. Valores elevados indicam semelhança entre a sub-janela e a característica Haar.

2) Imagens integrais: O valor das características Haar pode ser calculado rapidamente utilizando uma representação da imagem denominada imagem integral. O valor de um pixel na imagem integral é definido como a soma dos valores dos pixels à esquerda e acima, inclusive, na imagem original.

3) Treinamento AdaBoost: O processo de treinamento utiliza AdaBoost para selecionar um subconjunto significativo de características. Classificadores fracos são treinados, através da otimização de um limiar de classificação, utilizando apenas uma característica de forma a minimizar falsos negativos. Ou seja, um classificador por característica. Classificadores fortes são então construídos ao combinar classificadores fracos.

4) Classificadores em cascata: A deteç̧ão é realizada através de classificadores em cascata. No primeiro estágio, todas as sub-janelas da imagem são aplicadas ao melhor classificador forte construído com um apenas um classificador fraco (ou característica). As sub-janelas não detectadas corretamente são definitivamente descartadas. As outras subjanelas são aplicadas ao classificador do segundo estágio, o qual foi construído combinando alguns classificadores fracos. O número de classificadores fracos combinados para construir o classificador forte aumenta conforme o avanço nos estágios. O processo se repete até que a meta geral de falsos positivos e taxa de deteçãa é atendida. O conjunto de sub-janelas detectadas no último estágio forma a imagem do objeto.

$\mathrm{O}$ algoritmo de Viola-Jones foi aplicado às 300 imagens de cada indivíduo. Isso resultou em 300 imagens de cada um dos elementos faciais (rosto, boca, nariz e olhos) por indivíduo. 


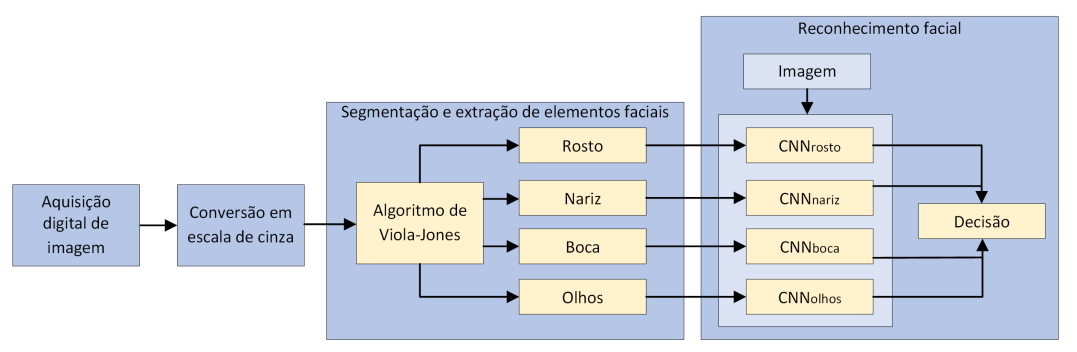

Fig. 1. Diagrama de fluxo da metodologia do algoritmo desenvolvido.

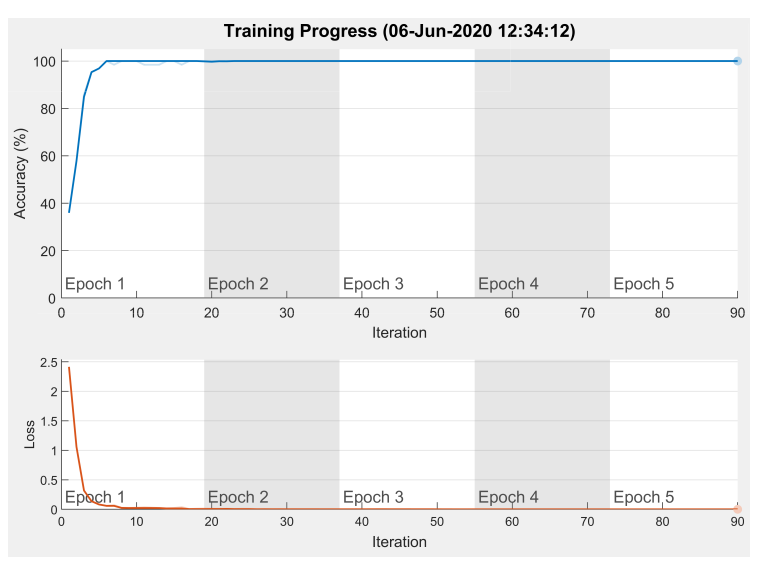

Fig. 2. Treinamento da $\mathrm{CNN}$ relativa ao rosto.

\section{Reconhecimento em tempo real}

A identificação dos indivíduos é realizada através de CNNs. As CNNs são utilizadas nos principais métodos do estado-daarte de reconhecimento facial [4]. Neste trabalho, optou-se por uma CNN para cada elemento facial, totalizando $4 \mathrm{CNNs}$, com $\mathrm{o}$ intuito de tornar o reconhecimento mais robusto.

Para cada elemento facial, as 300 imagens de cada indivíduo foram utilizadas como pares entrada/saída para treinar a correspondente $\mathrm{CNN}$ com validação cruzada. Utilizou-se uma validação cruzada estratificada em 5 vezes, onde os dados de treino são divididos em 5 grupos. Por época, 20\% dos dados de treino são alocados para validação cruzada de forma a validar o modelo. O algoritmo utilizado foi o SGDM (stochastic gradient descent with momentum), com taxa inicial de aprendizagem de 0,01 e funções de ativação ReLU (rectified linear unit) para todas as camadas, exceto na última onde foi utilizada a função de ativação softmax. O tamanho do kernel e o número de filtros são obtidos pela técnica de força bruta.

A decisão final do reconhecimento é baseada nas saídas das CNNs. Caso um indivíduo seja identificado por, pelo menos, 3 CNNs, ele é reconhecido pelo algoritmo. Caso contrário, assume-se que não houve reconhecimento do indivíduo.

\section{RESUltados E Discuss ÃO}

Os resultados de treinamento da CNN relativa ao rosto são exibidos na Figura 2. Resultados muito similares foram obtidos para as outras CNNs. Observa-se que uma acurácia de $100 \%$ é atingida após as primeiras iterações, indicando que as CNNs se ajustam completa e rapidamente aos dados.

Testes do reconhecimento em tempo real foram realizados. A Figura 3 ilustra o reconhecimento correto de Diana e Maria, autoras do trabalho. Note que o nome dos indivíduos é exibido no retângulo amarelo acima do rosto.

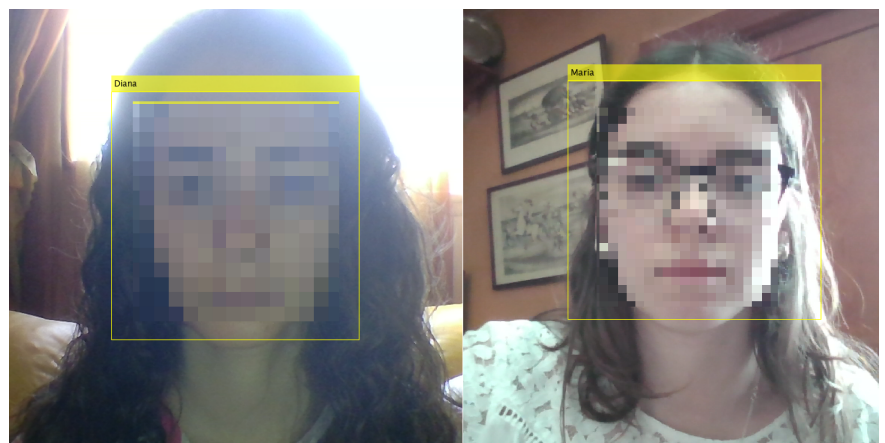

Fig. 3. Exemplos do algoritmo funcionando em tempo real.

Exemplos do algoritmo funcionando em tempo real estão disponíveis em https://drive.google.com/file/d/ 1 Hp 6m0 P cuMKc 6P QmWJyVOxLt Tqy cvn $2 x N$.

Algumas limitações do algoritmo desenvolvido foram notadas. O algoritmo de Viola-Jones necessita de otimização para diminuir os falsos positivos porque, às vezes, detecta mais elementos faciais que os corretos. Há necessidade de proximidade entre a câmera e o individuo para identificação. Movimentos bruscos dos indivíduos provocam erros de identificação.

\section{CONClus Ão}

Este trabalho apresentou os resultados preliminares do desenvolvimento de um algoritmo de reconhecimento facial em tempo real. Elementos faciais, nomeadamente, rosto, boca, nariz e olhos, são obtidos pelo algoritmo de Viola-Jones. Cada elemento facial é utilizado para treinar uma CNN. Apesar de resultados preliminares promissores, onde a prova de conceito foi conseguida, é necessário aumentar a base de imagens para realizar um rigoroso procedimento de treinamento e teste do algoritmo. Além disso, algumas melhorias no algoritmo são necessárias para efetiva aplicação em tempo real.

\section{AgRAdecimentos}

Esse trabalho foi apoiado pelos Fundos Nacionais da Fundação para a Ciência e a Tecnologia (FCT), Portugal, através do projeto UIDB/50016/2020.

\section{REFERÊNCIAS}

[1] J. B. Alam, M. M. Islam, T. Jabid, and S. Ahmed, "System development using face recognition," in Proceedings of the International Conference on Automation, Computational and Technology Management, April 2019.

[2] P. Viola and M. J. Jones, "Robust real-time face detection," International Journal of Computer Vision, vol. 57, no. 2, pp. 137-154, May 2004.

[3] - "Rapid object detection using a boosted cascade of simple features," in Proceedings of the IEEE Computer Society Conference on Computer Vision and Pattern Recognition., December 2001, pp. 511-518.

[4] S. Balaban, "Deep learning and face recognition: the state of the art," in Proceeding of XII Conference on Biometric and Surveillance Technology for Human and Activity Identification, 2015. 